\title{
Fatal renal failure as the first manifestation of sarcoidosis diagnosed on necropsy in a young man: a case report
}

\author{
A Awasthi, R Nada, P Malhotra, R Goel, K Joshi
}

J Clin Pathol 2004;57:1101-1103. doi: 10.1136/jicp.2004.018325

Renal involvement as the first manifestation of sarcoidosis is rare and has never been reported in India. This report describes a 35 year old man who was admitted to the emergency department with a clinical diagnosis of acute on chronic renal failure, secondary to obstructive uropathy. Postmortem examination unexpectedly revealed disseminated sarcoidosis.

A 35 year old man presented to the emergency department with a one week history of oliguria and altered sensorium. There was no history of renal colic, haematuria, or dysuria and there were no respiratory complaints in the past. On examination, he had bilateral "wet" crackles suggestive of fluid overload and a biphasic pericardial rub. There was no lymphadenopathy or hepatosplenomegaly.

Investigations revealed severe dimorphic anaemia (haemoglobin, $44 \mathrm{~g} /$ litre), leucocytosis (total leucocyte count, $14.4 \times 10^{9} /$ litre), and a raised erythrocyte sedimentation rate (82 mm first hour). Blood urea and serum creatinine were raised at $400 \mathrm{mg} \%$ and $9.7 \mathrm{mg} \%$, respectively. Urine examination revealed $1+$ albuminuria, pyuria (8-10 pus cells/high power field), and microhaematuria (8-10 red blood cells/ high power field). The kidneys were normal in size (right, $9.7 \mathrm{~cm}$; left, $9.9 \mathrm{~cm}$ ) on ultrasonography. However, there was an indistinct corticomedullary differentiation, along with bilateral hydroureteronephrosis and left sided nephrolithiasis. Biochemical analysis revealed normal serum sodium (130 and $133 \mathrm{mg} \%$, respectively) and potassium (3 and $5.2 \mathrm{mg} \%$, respectively) values. Inorganic phosphate was raised ( $11.7 \mathrm{mg} \%)$, whereas serum calcium was low to normal ( $8.8 \mathrm{mg} \%$ ). Liver function tests and total proteins (60 g/litre; albumin to globulin ratio of 1.0 ) were within normal limits. Arterial blood gases revealed metabolic acidosis and there was evidence of pulmonary oedema on chest $x$ ray. Pus from a $2 \times 1.5 \mathrm{~cm}$ sized perianal abscess grew Escherichia coli sensitive to amikacin. Blood and urine cultures were sterile. The patient was treated with intravenous cloxacillin and amikacin at renal modified doses. Eighteen cycles of peritoneal dialysis were carried out; however, he had an episode of ventricular fibrillation and died.

A complete necropsy was performed after informed consent from the relatives. Both kidneys were normal in size (left, $9.7 \mathrm{~cm}$; right, $9.5 \mathrm{~cm}$ ) with evidence of bilateral hydroureteronephrosis. There was a $3 \mathrm{~cm}$ diameter stone in the left renal pelvis and a $2 \mathrm{~cm}$ diameter stone in the lower part of the left ureter. Microscopic examination revealed pronounced nephrocalcinosis in the form of calcification of the basement membranes and tubular epithelium, along with the interstitium (fig 1). In addition, there were focal masses of calcification surrounded by polymorphs. Calcification of the smooth muscles and the intima of medium sized blood vessels and glomeruli (fig 1, inset) was also present. The lungs were subcrepitant and there were enlarged hilar lymph nodes $(2.5 \times 4 \mathrm{~cm}$ in diameter $)$. On microscopy of the lungs, typical non-caseating epithelioid cell granulomas were seen (fig 2). Metastatic calcification was seen in the alveolar septae and blood vessels. The hilar lymph nodes showed diffuse paracortical sclerosis and the presence of fibrosed granulomas in the cortex. Giant cells in these granulomas revealed asteroid bodies. The liver was normal on gross examination. However, microscopically there were stellate scars connecting portal tracts and central veins. Some of these scars were composed of healed epithelioid cell granulomas with giant cells containing asteroid bodies (fig 3). Similar lesions were seen in the spleen. There was no evidence of necrosis in the granulomas. Metastatic calcification was also present in the duramater, myocardium, and in the vessels of the gastrointestinal tract and brain.

Therefore, the final postmortem diagnosis was sarcoidosis involving the lung, hilar lymph nodes, liver, and spleen with renal involvement in the form of nephrocalcinosis, hydroureteronephrosis secondary to nephrolithiasis, and chronic interstitial nephritis. There was also evidence of widespread metastatic calcification in the duramater, myocardium, and the blood vessels of lung, gastrointestinal tract, and brain.

\section{DISCUSSION}

Sarcoidosis, a disease that is increasingly being recognised in India, primarily involves the lungs and lymphatic system. ${ }^{12}$ Renal involvement is less frequent; the incidence in the West ranges from $7 \%$ to $22 \%{ }^{3}$ whereas two large

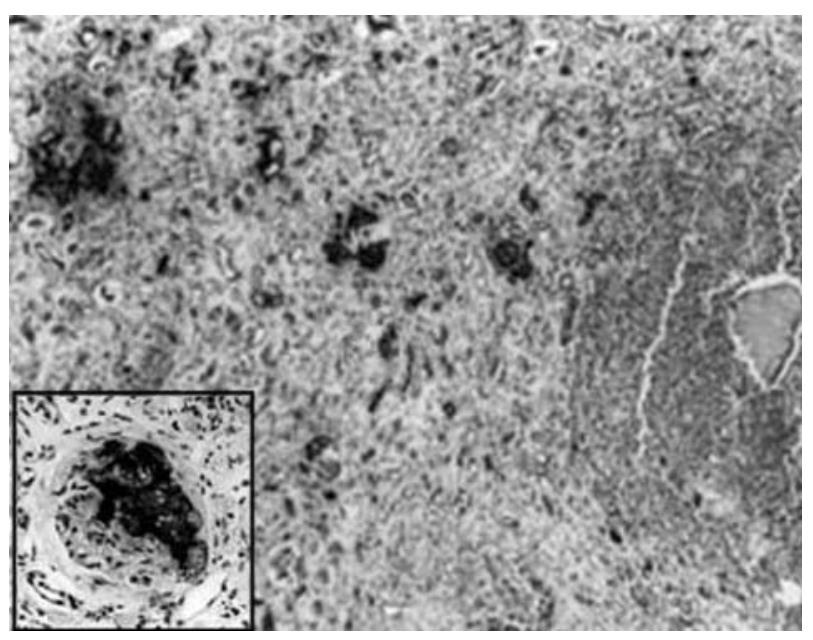

Figure 1 Pronounced nephrocalcinosis in the form of interstitial and tubular calcification and a calcified mass within an abscess (right). Haematoxylin and eosin (H\&E) staining; original magnification, $\times 13.2$. Inset: glomerular calcification. H\&E staining; original magnification, $\times 66$. 


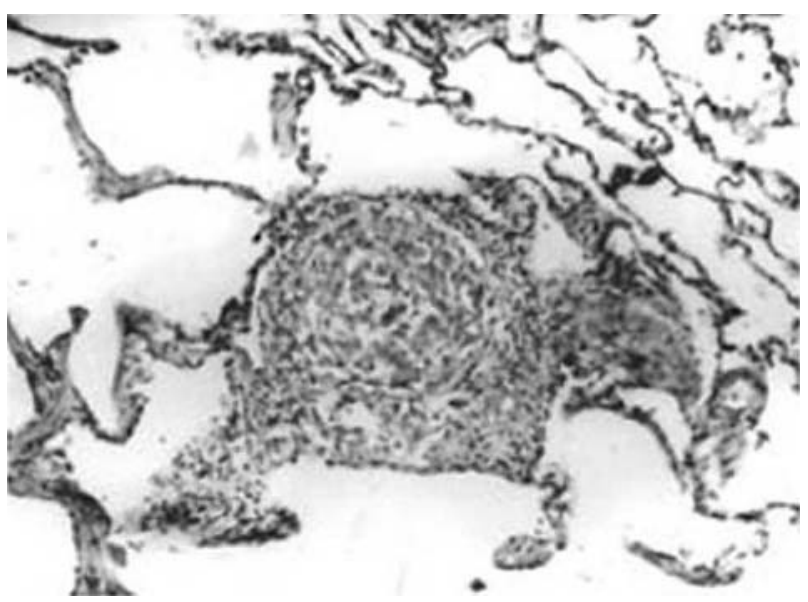

Figure 2 Photomicrograph showing a compact epithelioid cell granuloma in the pulmonary interstitium. Haematoxylin and eosin staining; original magnification, $\times 33$.

studies from India failed to identify renal involvement as the presenting feature in their patients. ${ }^{45}$ Morphologically, involvement of the kidneys in sarcoidosis can occur in three ways: nephrocalcinosis and nephrolithiasis, direct granulomatous involvement of the tubulo-interstitium, or glomerulonephritis. ${ }^{6}$

"The pathophysiological mechanisms responsible for renal failure in sarcoidosis include a direct effect of hypercalcaemia, dehydration, nephrocalcinosis, and tubulointerstitial disease"

In a large retrospective study by Rizzato et al renal calculi, usually composed of calcium oxalate crystals, were the first manifestation of disease in $2.2 \%$ of their patients, although unlike our present case, none of the patients in that series presented with renal failure. ${ }^{7}$ In our case, in addition to the large left sided renal and ureteric calculi there was pronounced tubular, vascular, and focal glomerular calcification.

The pathophysiological mechanisms responsible for renal failure in sarcoidosis include a direct effect of hypercalcaemia, dehydration, nephrocalcinosis, and tubulointerstitial disease. ${ }^{1}$ Most of these factors probably contributed to the renal failure in our patient. In contrast to the cases of renal sarcoidosis reported by Ohashi and colleagues ${ }^{8}$ and Fernandez Giron et al, ${ }^{9}$ where hypercalcaemia accompanied renal failure, the index case had normal calcium values at presentation. However, hypercalcaemia in the past probably contributed to the renal failure. This was evident from the widespread metastatic calcification including severe nephrocalcinosis seen at necropsy, which suggests the presence of at least moderate to severe hypercalcaemia in the past. In fact, the presence of metastatic calcification usually correlates with serum calcium concentration over $13 \mathrm{mg} \%{ }^{10}$ The low to normal serum calcium in our patient is also attributable to the presence of advanced renal failure. In renal failure, reduced excretion of phosphate and decreased production of $1,25(\mathrm{OH})_{2}$ vitamin $\mathrm{D}_{3}$ result in reduced skeletal responsiveness to parathyroid hormone. The $\mathrm{l}$, $25(\mathrm{OH})_{2}$ vitamin $\mathrm{D}_{3}$ deficiency also interferes with calcium absorption from the gut, which is already impaired in uraemia.

In conclusion, our case shows that renal failure resulting in death can rarely be the initial manifestation of sarcoidosis. The presence of normal serum calcium values in a patient

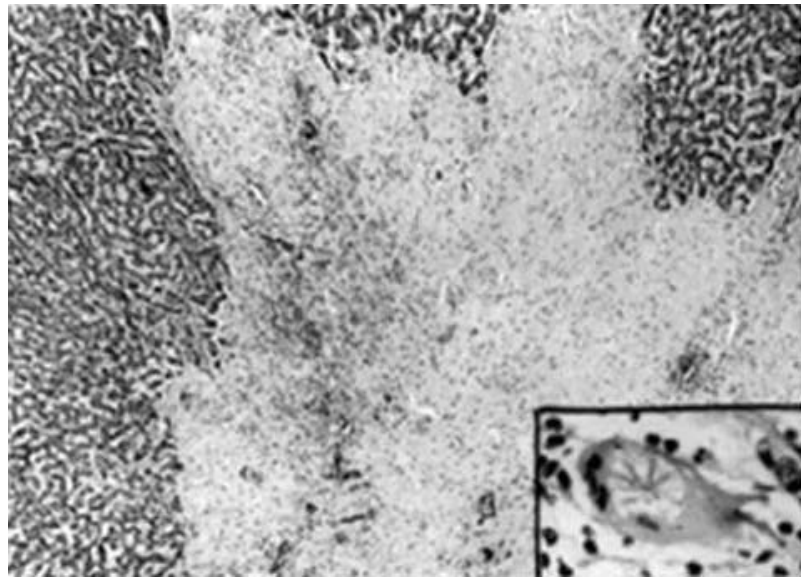

Figure 3 Large stellate areas of fibrosis in the portal tracts with scattered inflammatory cells and a giant cell containing an asteroid body. Haematoxylin and eosin staining; original magnification, $\times 13.2$.

\section{Take home messages}

- Renal failure resulting in death can rarely be the initial manifestation of sarcoidosis

- We report the first case of sarcoidosis in India presenting as fatal renal failure

- The presence of normal serum calcium in a patient with nephrolithiasis, nephrocalcinosis, and advanced renal failure does not exclude sarcoidosis as a possible cause because calcium concentrations may be normalised as a result of secondary hyperparathyroidism of renal failure or widespread metastatic deposition of calcium

with nephrolithiasis, nephrocalcinosis, and advanced renal failure does not exclude sarcoidosis as a possible cause; calcium concentrations may be normalised as a result of secondary hyperparathyroidism of renal failure or widespread metastatic deposition of calcium.

\section{ACKNOWLEDGEMENTS}

We are grateful to Professor SK Jindal, Head of the Department of Pulmonary Medicine, PGIMER, Chandigarh for providing guidance and data on sarcoidosis in India during the preparation of this manuscript.

\section{Authors' affiliations}

A Awasthi, R Nada, R Goel, K Joshi, Department of Histopathology, Postgraduate Institute of Medical Education and Research, Chandigarh160012, India

P Malhotra, Department of Pulmonary Medicine, Postgraduate Institute of Medical Education and Research

Correspondence to: Dr A Awasthi, Department of Pathology, Postgraduate Institute of Medical Education and Research, Chandigarh160012, India; dranshupuneet@yahoo.com

Accepted for publication 13 April 2004

\section{REFERENCES}

1 Hunninghake GW, Constable $U$, Ando $M$, et al. Statement on sarcoidosis. Am J Respir Crit Care Med 1999;160:736-55.

2 Jindal SK, Gupta D, Aggarwal AN. Sarcoidosis in India: practical issues and difficulties in diagnosis and management. Sarcoidosis Vasc Diffuse Lung Dis 2002; 19:176-84.

3 MacSearraigh ET, Doyle CT, Twomey M, et al. Sarcoidosis with renal involvement. Postgrad Med J 1978;54:528-32. 
4 Sharma R, Guleria R, Mohan A, et al. Clinical profile of 153 patients with biopsy proven sarcoidosis. Results from a centre in India [abstract]. J Assoc Physicians India 2003;51:1201

5 Bambery P, Behera D, Gupta AK, et al. Sarcoidosis in north India: the clinical profile of 40 patients. Sarcoidosis 1987:4:155-8.

6 Akmal M, Sharma OP. Renal sarcoidosis; a reminder. Chest 1990;97:1284-5

7 Rizzato G, Fraioli P, Montemurro L. Nephrolithiasis as a presenting feature of chronic sarcoidosis. Thorax 1995;50:555-9.
8 Ohashi $\mathrm{N}$ Yonemura $\mathrm{K}$, Hirano $\mathrm{M}$ et al A patient with sarcoidosis presenting with acute renal failure: implication for granulomatous interstitial nephritis and hypercalcemia. Intern Med 2002;41:1 171-4.

9 Fernandez Giron F, Fernandez Mora F, Conde-Garcia J. Granulomatous interstitial nephritis, hypercalcemia and rapidly progressive kidney failure secondary to sarcoidosis with exclusive renal involvement. Am J Nephrol 2001;21:514-16.

10 Haviv YS, Silver J. Hypercalcemia. In: Wass JAH, Shalet SM, eds. Oxford textbook of endocrinology and diabetes, 1 st ed. Oxford University Press, 2002:609-17. 